\title{
Measurements of mass, width and gauge couplings of the W boson at LEP
}

\section{L3 Collaboration}

M. Acciarri ${ }^{\text {ac }}$, O. Adriani ${ }^{\mathrm{r}}$, M. Aguilar-Benitez ${ }^{\mathrm{ab}}$, S. Ahlen ${ }^{~}$, J. Alcaraz ${ }^{\mathrm{ab}}$,

G. Alemanni ${ }^{x}$, J. Allaby ${ }^{\text {s }}$, A. Aloisio ${ }^{\text {ae }}$, G. Alverson ${ }^{\mathrm{m}}$, M.G. Alviggi ${ }^{\text {ae }}$, G. Ambrosi ", H. Anderhub ay , V.P. Andreev g,an , T. Angelescu " , F. Anselmo ${ }^{j}$, A. Arefiev ${ }^{\text {ad }}$, T. Azemoon ${ }^{\text {c }}$, T. Aziz ${ }^{\text {k }}$, P. Bagnaia am ${ }^{\text {a }}$ L. Baksay ${ }^{\text {at }}$, S. Banerjee ${ }^{k}$, Sw. Banerjee ${ }^{\mathrm{k}}$, K. Banicz av ${ }^{\text {a }}$ A. Barczyk ${ }^{\text {ay,aw }}$, R. Barillère ${ }^{\mathrm{s}}$, L. Barone ${ }^{\text {am }}$, P. Bartalini aj, A. Baschirotto ${ }^{\text {ac }}$, M. Basile ${ }^{\mathrm{j}}$, R. Battiston ${ }^{\text {aj }}$, A. Bay ${ }^{\mathrm{x}}$, F. Becattini ${ }^{\mathrm{r}}$, U. Becker ${ }^{\mathrm{q}}$, F. Behner ${ }^{\text {ay }}$, J. Berdugo ${ }^{\text {ab }}$, P. Berges ${ }^{\mathrm{q}}$, B. Bertucci ${ }^{\text {aj }, ~ B . L . ~ B e t e v ~}{ }^{\text {ay }}$,

S. Bhattacharya ${ }^{\mathrm{k}}$, M. Biasini ${ }^{\mathrm{s}}$, A. Biland ${ }^{\mathrm{ay}}$, G.M. Bilei aj, J.J. Blaising ${ }^{\mathrm{d}}$, S.C. Blyth ${ }^{\text {ak }}$, G.J. Bobbink ${ }^{b}$, R. Bock ${ }^{a}$, A. Böhm ${ }^{a}$, L. Boldizsar ${ }^{\circ}$, B. Borgia ${ }^{\text {am }}$, D. Bourilkov ay , M. Bourquin ", S. Braccini ", J.G. Branson ${ }^{\text {ap }, ~ V . ~ B r i g l j e v i c ~ a y, ~}$ I.C. Brock ${ }^{\text {ak }}$, A. Buffini ${ }^{\text {r }}$ A. Buijs ${ }^{\text {au }}$, J.D. Burger ${ }^{q}$, W.J. Burger " , J. Busenitz ${ }^{\text {at }}$, A. Button ${ }^{\text {c }}$, X.D. Cai ${ }^{\mathrm{q}}$, M. Campanelli ay, M. Capell ${ }^{\mathrm{q}}$, G. Cara Romeo ${ }^{\mathrm{j}}$, G. Carlino ${ }^{\text {ae }}$, A.M. Cartacci ${ }^{\mathrm{r}}$, J. Casaus ${ }^{\text {ab }}$, G. Castellini ${ }^{\mathrm{r}}$, F. Cavallari ${ }^{\mathrm{am}}$, N. Cavallo ${ }^{\text {ac }}$, C. Cecchi ${ }^{\text {u, }}$ M. Cerrada ${ }^{\text {ab }}$, F. Cesaroni ${ }^{y}$, M. Chamizo ${ }^{\text {ab }}$, Y.H. Chang ${ }^{\text {ba }}$, U.K. Chaturvedi ${ }^{\text {t }}$ S.V. Chekanov ${ }^{\text {ag }}$, M. Chemarin ${ }^{\text {aa }}$, A. Chen ${ }^{\text {ba }}$, G. Chen ${ }^{\text {h }}$, G.M. Chen ${ }^{\text {h }}$, H.F. Chen ${ }^{\mathrm{v}}$, H.S. Chen ${ }^{\mathrm{h}}$, X. Chereau ${ }^{\mathrm{d}}$, G. Chiefari ${ }^{\text {ae }}$, C.Y. Chien ${ }^{\text {e }}$, L. Cifarelli ao , F. Cindolo ${ }^{\text {j }}$, C. Civinini ${ }^{\text {r }}$, I. Clare ${ }^{\mathrm{q}}$, R. Clare ${ }^{\mathrm{q}}$, H.O. Cohn ${ }^{\text {ah }}$, G. Coignet ${ }^{d}$, A.P. Colijn ${ }^{b}$, N. Colino ${ }^{\text {ab }}$, V. Commichau ${ }^{a}$, S. Costantini i ${ }^{\text {, F F Cotorobai }}{ }^{n}$, B. de la Cruz ${ }^{\text {ab }}$, A. Csilling ${ }^{\circ}$, T.S. Dai ${ }^{\text {, }}$, R. D'Alessandro ${ }^{\text {r }}$, R. de Asmundis ${ }^{\text {ae }}$, A. Degré ${ }^{\mathrm{d}}$, K. Deiters ${ }^{\text {aw }}$, D. della Volpe ${ }^{\text {ae }}$, P. Denes ${ }^{\text {al }}$, F. DeNotaristefani ${ }^{\text {am }}$, D. DiBitonto ${ }^{\text {at }}, \mathrm{M}$. Diemoz ${ }^{\text {am }}$, D. van Dierendonck ${ }^{b}$, F. Di Lodovico ay, C. Dionisi ${ }^{a m}$, M. Dittmar ${ }^{\text {ay }}$, A. Dominguez ap , A. Doria ${ }^{\text {ae }}$, M.T. Dova ${ }^{\mathrm{t}, 1}$, D. Duchesneau ${ }^{\mathrm{d}}$, P. Duinker ${ }^{\mathrm{b}}$, I. Duran ${ }^{\text {aq }}$, S. Dutta ${ }^{\text {}}$, S. Easo ${ }^{\text {aj }}$, Yu. Efremenko ${ }^{\text {ah }}$, H. El Mamouni ${ }^{\text {aa }}$, A. Engler ak, F.J. Eppling ${ }^{\mathrm{q}}$, F.C. Erné ${ }^{\text {b }}$, J.P. Ernenwein ${ }^{\text {aa }}$, P. Extermann ${ }^{\text {u }}$, M. Fabre ${ }^{\text {aw }}$, R. Faccini ${ }^{\text {am }}$, S. Falciano ${ }^{\text {am }}$, A. Favara ${ }^{\mathrm{r}}$, J. Fay ${ }^{\text {aa }}$, O. Fedin ${ }^{\text {an }}$, M. Felcini ${ }^{\text {ay }}$, 
B. Fenyi ${ }^{\text {at }}$, T. Ferguson ${ }^{\text {ak }}$, F. Ferroni ${ }^{\text {am }}$, H. Fesefeldt ${ }^{a}$, E. Fiandrini ${ }^{\text {aj }}$, J.H. Field ${ }^{\text {u, }}$, F. Filthaut ${ }^{\text {ak }}$, P.H. Fisher ${ }^{q}$, I. Fisk ${ }^{\text {ap }}$, G. Forconi ${ }^{q}$, L. Fredj ", K. Freudenreich ay, C. Furetta ${ }^{\text {ac }}$, Yu. Galaktionov ad, , S.N. Ganguli ${ }^{k}$, P. Garcia-Abia ${ }^{\text {ax }}$, S.S. Gau ${ }^{\mathrm{m}}$, S. Gentile ${ }^{\text {am }}$, N. Gheordanescu ${ }^{\mathrm{n}}$, S. Giagu ${ }^{\text {am }}$, S. Goldfarb ${ }^{\mathrm{x}}$, J. Goldstein ${ }^{\mathrm{I}}$, Z.F. Gong v ${ }^{\text {v }}$ A. Gougas ${ }^{\mathrm{e}}$, G. Gratta ai , M.W. Gruenewald ${ }^{\mathrm{i}}$, V.K. Gupta al , A. Gurtu ${ }^{\text {k }}$, L.J. Gutay av, B. Hartmann ${ }^{\text {a }}$, A. Hasan af ${ }^{\text {, D. Hatzifotiadou }}$ j, T. Hebbeker ${ }^{\text {i }}$, A. Hervé s, W.C. van Hoek ${ }^{\text {ag }}$, H. Hofer ay, S.J. Hong ${ }^{\text {as }}$, H. Hoorani ${ }^{\text {ak }}$, S.R. Hou ba, G. Hu ${ }^{\text {e }}$, V. Innocente ${ }^{\text {s }}$, K. Jenkes ${ }^{a}$, B.N. Jin ${ }^{\text {h }}$, L.W. Jones ${ }^{c}$, P. de Jong ${ }^{\text {s, }}$ I. Josa-Mutuberria ${ }^{\text {ab }}$, A. Kasser ${ }^{\text {x }}$, R.A. Khan ${ }^{\text {t}}$, D. Kamrad ax , Yu. Kamyshkov ah, J.S. Kapustinsky ${ }^{\text {z }}$, Y. Karyotakis ${ }^{d}$, M. Kaur ${ }^{\text {t, }}$, M.N. Kienzle-Focacci ${ }^{\text {u, D. Kim }}{ }^{\text {am }}$, D.H. Kim as, J.K. Kim ${ }^{\text {as }}$, S.C. Kim ${ }^{\text {as }}$, Y.G. Kim ${ }^{\text {as }}$, W.W. Kinnison ${ }^{\text {z }}$, A. Kirkby ai D. Kirkby ${ }^{\text {ai }}$, J. Kirkby ${ }^{\text {s, D. Kiss }}{ }^{\circ}$, W. Kittel ${ }^{\text {ag }}$, A. Klimentov ${ }^{\text {q,ad }}$, A.C. König ag, A. Kopp ax , I. Korolko ad, V. Koutsenko ${ }^{\text {q,ad }}$, R.W. Kraemer ${ }^{\text {ak }}$, W. Krenz ${ }^{\text {a }}$, A. Kunin q,ad, P. Ladron de Guevara ab ${ }^{\text {ab }}$ I. Laktineh aa, G. Landi ' , C. Lapoint ${ }^{q}$, K. Lassila-Perini ay, P. Laurikainen " ${ }^{\text {w }}$ M. Lebeau ${ }^{\text {s, }}$ A. Lebedev ${ }^{\mathrm{q}}$, P. Lebrun aa, P. Lecomte ${ }^{\text {ay }}$, P. Lecoq ${ }^{\text {s }}$ P. Le Coultre ${ }^{\text {ay }}$, H.J. Lee ${ }^{\text {i }}$, J.M. Le Goff ${ }^{\text {s }}$, R. Leiste ${ }^{\text {ax }}$, E. Leonardi am, P. Levtchenko an, C. Li v ${ }^{\text {v }}$ C.H. Lin ${ }^{\text {ba }}$, W.T. Lin ${ }^{\text {ba }}$, F.L. Linde ${ }^{\text {b,s }}$, L. Lista ${ }^{\text {ae }}$, Z.A. Liu ${ }^{\text {h}}$, W. Lohmann ${ }^{\text {ax }}$, E. Longo ${ }^{\text {am }}$, W. Lu ${ }^{\text {ai }}$, Y.S. Lu ${ }^{\text {he }}$, K. Lübelsmeyer ${ }^{a}$, C. Luci ${ }^{\text {am }}$, D. Luckey ${ }^{\mathrm{q}}$, L. Luminari am, W. Lustermann ${ }^{\text {aw }}$, W.G. Ma ${ }^{v}$, M. Maity ${ }^{k}$, G. Majumder ${ }^{\text {k }}$, L. Malgeri ${ }^{a m}$, A. Malinin ${ }^{\text {ad }}$, C. Maña ${ }^{a b}$, D. Mangeol ag, S. Mangla ${ }^{\text {k}}$, P. Marchesini ay , A. Marin ${ }^{~}$, J.P. Martin aa, F. Marzano ${ }^{\text {am }}$, G.G.G. Massaro ${ }^{\text {b }}$, D. McNally ${ }^{~}$, R.R. McNeil ${ }^{\mathrm{g}}$, S. Mele ${ }^{\text {ae }}$, L. Merola ${ }^{\text {ae }}$, M. Meschini ${ }^{\mathrm{I}}$, W.J. Metzger ag , M. von der Mey ${ }^{\mathrm{a}}$, Y. Mi ${ }^{\mathrm{x}}$, A. Mihul ${ }^{\mathrm{n}}$, A.J.W. van Mil ag, H. Milcent ${ }^{\text {s}}$, G. Mirabelli ${ }^{\text {am }}$, J. Mnich ${ }^{\text {s }}$, P. Molnar ${ }^{\text {i }}$, B. Monteleoni ${ }^{\mathrm{r}}$, R. Moore ${ }^{\mathrm{c}}$, S. Morganti ${ }^{\text {am }}$, T. Moulik ${ }^{\mathrm{k}}$, R. Mount ${ }^{\text {ai }}$, S. Müller ${ }^{\text {a }}$, F. Muheim ", A.J.M. Muijs ${ }^{\text {b }}$, S. Nahn ${ }^{\text {q }}$, M. Napolitano ${ }^{\text {ae }}$, F. Nessi-Tedaldi ay, H. Newman ai , T. Niessen ${ }^{a}$, A. Nippe ${ }^{\text {a }}$, A. Nisati ${ }^{\text {am }}$, H. Nowak ${ }^{\text {ax }}$, Y.D. Oh ${ }^{\text {as }}$, H. Opitz ${ }^{\text {a }}$, G. Organtini am , R. Ostonen ", C. Palomares ${ }^{\text {ab }}$, D. Pandoulas ${ }^{\text {a }}$, S. Paoletti ${ }^{\text {am }}$, P. Paolucci ae , H.K. Park ${ }^{\text {ak }}$, I.H. Park ${ }^{\text {as }}$, G. Pascale am , G. Passaleva ${ }^{\text {s }}$, S. Patricelli ae, T. Paul ${ }^{\mathrm{m}}$, M. Pauluzzi aj, C. Paus ${ }^{\mathrm{s}}$, F. Pauss ${ }^{\text {ay }}$, D. Peach ${ }^{\mathrm{s}}$, Y.J. Pei ${ }^{a}$, S. Pensotti ${ }^{\text {ac }}$, D. Perret-Gallix ${ }^{\text {d }}$, B. Petersen ${ }^{\text {ag }}$, S. Petrak ${ }^{\text {i }}$, A. Pevsner ${ }^{\text {e, }}$ D. Piccolo ${ }^{\text {ae }}$, M. Pieri ${ }^{\text {T }}$ P.A. Piroué al, E. Pistolesi ac, V. Plyaskin ad, M. Pohl ay, V. Pojidaev ${ }^{\text {ad, }, \text {, H. Postema }}{ }^{\text {q, N. Produit ", D. Prokofiev an , G. Rahal-Callot ay }}$, N. Raja ${ }^{\text {k}}$, P.G. Rancoita ${ }^{a c}$, M. Rattaggi ${ }^{\text {ac }}$, G. Raven ap , P. Razis ${ }^{\text {af }}$, K. Read ${ }^{\text {ah }}$, D. Ren ${ }^{\text {ay }}$, M. Rescigno ${ }^{\text {am }}$, S. Reucroft ${ }^{\mathrm{m}}$, T. van Rhee ${ }^{\text {au }}$, S. Riemann ${ }^{\text {ax }}$, K. Riles ${ }^{\text {c }}$, A. Robohm ay, J. Rodin ${ }^{\text {q, }}$ B.P. Roe ${ }^{\text {c }}$, L. Romero ${ }^{\text {ab }}$, S. Rosier-Lees ${ }^{\text {d }}$, Ph. Rosselet ${ }^{\text {X }}$, W. van Rossum au, S. Roth ${ }^{\text {a }}$, J.A. Rubio ${ }^{\text {s }}$, D. Ruschmeier ${ }^{\text {i }}$, 
H. Rykaczewski ay , J. Salicio ${ }^{\text {s }}$, E. Sanchez ${ }^{\text {ab }}$, M.P. Sanders ${ }^{\text {ag }}$, M.E. Sarakinos ${ }^{\text {w }}$, S. Sarkar k, M. Sassowsky a , C. Schäfer a , V. Schegelsky ${ }^{\text {an }}$, S. Schmidt-Kaerst ${ }^{\text {a }}$, D. Schmitz a , P. Schmitz a, N. Scholz ay , H. Schopper ${ }^{\text {az }}$, D.J. Schotanus ${ }^{\text {ag }}$, J. Schwenke ${ }^{\text {a }}$, G. Schwering a , C. Sciacca ${ }^{\text {ae }}$, D. Sciarrino ", L. Servoli aj, S. Shevchenko ${ }^{\text {ai }}$, N. Shivarov ${ }^{\text {ar }}$, V. Shoutko ${ }^{\text {ad }}$, J. Shukla ${ }^{z}$, E. Shumilov ${ }^{\text {ad }}$, A. Shvorob ai, T. Siedenburg ${ }^{\text {a }}$, D. Son ${ }^{\text {as }}$, A. Sopczak ${ }^{\text {ax }}$, B. Smith ${ }^{\text {q }}$, P. Spillantini ${ }^{\text {r }}$, M. Steuer ${ }^{\text {q }}$, D.P. Stickland al, A. Stone ${ }^{\mathrm{g}}$, H. Stone ${ }^{\text {al }}$, B. Stoyanov ${ }^{\text {ar }}$, A. Straessner ${ }^{\text {a }}$, K. Strauch ${ }^{\text {p }}$, K. Sudhakar ${ }^{k}$, G. Sultanov ${ }^{\mathrm{t}}$, L.Z. Sun ${ }^{\text {v }}$, G.F. Susinno ", H. Suter ${ }^{\text {ay }}$, J.D. Swain ${ }^{\mathrm{t}}$, X.W. Tang ${ }^{\mathrm{h}}$, L. Tauscher ${ }^{\mathrm{f}}$, L. Taylor ${ }^{\mathrm{m}}$, Samuel C.C. Ting ${ }^{\mathrm{q}}$, S.M. Ting ${ }^{\mathrm{q}}$, M. Tonutti ${ }^{\text {a }}$, S.C. Tonwar ${ }^{\mathrm{k}}$, J. Tóth ${ }^{\circ}$, C. Tully ${ }^{\text {al }}, \mathrm{H}$. Tuchscherer ${ }^{\text {at }}$, K.L. Tung ${ }^{\text {h }}$, Y. Uchida ${ }^{\text {q }}$, J. Ulbricht ${ }^{\text {ay }}$, U. Uwer ${ }^{\text {s }}$, E. Valente ${ }^{\text {am }}$, R.T. Van de Walle ${ }^{\text {ag }}$, G. Vesztergombi ${ }^{\circ}$, I. Vetlitsky ${ }^{\text {ad }}$, G. Viertel ${ }^{\text {ay }}$, M. Vivargent ${ }^{d}$, R. Völkert ${ }^{\text {ax }}$, H. Vogel ${ }^{\text {ak }}$, H. Vogt ${ }^{\text {ax }}$, I. Vorobiev ${ }^{\text {s,ad }}$, A.A. Vorobyov ${ }^{\text {an }}$, A. Vorvolakos ${ }^{\text {af }}$, M. Wadhwa ${ }^{\text {f }}$, W. Wallraff ${ }^{\text {a }}$, J.C. Wang ${ }^{q}$, X.L. Wang " , Z.M. Wang " , A. Weber ${ }^{\mathrm{a}}$, F. Wittgenstein ${ }^{\mathrm{s}}$, S.X. Wu ${ }^{\mathrm{t}}$, S. Wynhoff ${ }^{\mathrm{a}}$, J. Xu ${ }^{1}$, Z.Z. Xu ${ }^{\text {v }, ~ B . Z . ~ Y a n g ~}{ }^{v}$, C.G. Yang ${ }^{\text {h }}$, X.Y. Yao ${ }^{\text {h }}$, J.B. Ye ${ }^{\text {v }, ~ S . C . ~ Y e h ~}{ }^{\text {ba }}$, J.M. You ${ }^{\text {ak }}$, An. Zalite ${ }^{\text {an }}$, Yu. Zalite ${ }^{\text {an }}$, P. Zemp ${ }^{\text {ay }}$, Y. Zeng ${ }^{\text {a }}$, Z. Zhang ${ }^{\text {h, }}$ Z.P. Zhang ${ }^{\text {v }}$, B. Zhou ${ }^{\text {l }}$, G.Y. Zhu ${ }^{\text {h}}$, R.Y. Zhu ${ }^{\text {ai }}$, A. Zichichi ${ }^{\text {j.s.t }}$, F. Ziegler ${ }^{\text {ax }}$

${ }^{a}$ I. Physikalisches Institut, RWTH, D-52056 Aachen, FRG ${ }^{3}$

III. Physikalisches Institut, RWTH, D-52056 Aachen, FRG ${ }^{3}$

${ }^{b}$ National Institute for High Energy Physics, NIKHEF, and University of Amsterdam, NL-1009 DB Amsterdam, The Netherlands ' University of Michigan. Ann Arbor, MI 48109, USA

${ }^{d}$ Laboratoire d'Annecy-le-Vieux de Physique des Particules, LAPP,IN2P3-CNRS, BP 110, F-7494I Annecy-le-Vieux CEDEX, France e Johns Hopkins University, Baltimore, MD 21218, USA

${ }^{\mathrm{f}}$ Institute of Physics, University of Basel, CH-4056 Basel, Switzerland

${ }^{\mathrm{g}}$ Louisiana State University, Baton Rouge, LA 70803, USA

${ }^{\mathrm{h}}$ Institute of High Energy Physics, IHEP, 100039 Beijing, China ${ }^{4}$ i Humboldt University, D-10099 Berlin, FRG ${ }^{3}$

${ }^{\mathrm{j}}$ University of Bologna and INFN-Sezione di Bologna, I-40126 Bologna, Italy

${ }^{k}$ Tata Institute of Fundamental Research, Bombay 400005 , India

'Boston University, Boston, MA 02215, USA

${ }^{\mathrm{m}}$ Northeastern University, Boston, MA 02115, USA

"Institute of Atomic Physics and University of Bucharest, R-76900 Bucharest, Romania

${ }^{\circ}$ Central Research Institute for Physics of the Hungarian Academy of Sciences, H-1525 Budapest 114, Hungary ${ }^{5}$

${ }^{\mathrm{p}}$ Harvard University, Cambridge, MA 02139, USA

${ }^{\mathrm{q}}$ Massachusetts Institute of Technology, Cambridge, MA 02139, USA

${ }^{\top}$ INFN Sezione di Firenze and University of Florence, I-50125 Florence, Italy

' European Laboratory for Particle Physics, CERN, CH-1211 Geneva 23, Switzerland

'World Laboratory. FBLJA Project, CH-1211 Geneva 23, Switzerland

"University of Geneva, CH-1211 Geneva 4, Switzerland

"Chinese University of Science and Technology, USTC, Hefei, Anhui 230029, China $^{4}$

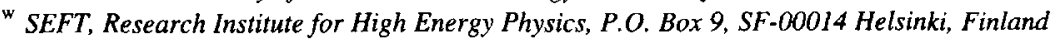

${ }^{x}$ University of Lausanne, CH-1015 Lausanne, Switzerland

y INFN-Sezione di Lecce and Universitá Degli Studi di Lecce, I-73100 Lecce, Italy

${ }^{2}$ Los Alamos National Laboratory, Los Alamos, NM 87544, USA

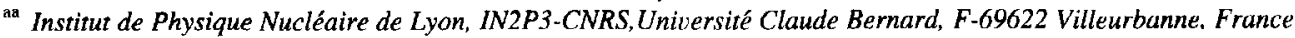


${ }^{\text {ab }}$ Centro de Investigaciones Energeticas, Medioambientales y Tecnologicas, CIEMAT, E-28040 Madrid, Spain ${ }^{6}$

ac INFN-Sezione di Milano, I-20133 Milan, Italy

ad Institute of Theoretical and Experimental Physics, ITEP, Moscow, Russia

ae INFN-Sezione di Napoli and University of Naples, I-80125 Naples, Italy

${ }^{\text {af }}$ Department of Natural Sciences, University of Cyprus, Nicosia, Cyprus

ag University of Nijmegen and NIKHEF, NL-6525 ED Nijmegen, The Netherlands

ah Oak Ridge National Laboratory, Oak Ridge, TN 37831, USA

ai California Institute of Technology, Pasadena, CA 91125, USA

aj INFN-Sezione di Perugia and Universitá Degli Studi di Perugia, I-06100 Perugia, Italy

ak Carnegie Mellon University, Pittsburgh, PA 15213, USA

at Princeton University, Princeton, NJ 08544, USA

am INFN-Sezione di Roma and University of Rome, "La Sapienza", I-00185 Rome, Italy

${ }^{\text {an }}$ Nuclear Physics Institute, St. Petersburg, Russia

ao University and INFN, Salerno, I-84100 Salerno, Italy

ap University of California, San Diego, CA 92093, USA

aq Dept, de Fisica de Particulas Elementales, Univ. de Santiago, E-15706 Santiago de Compostela, Spain

${ }^{a}$ Bulgarian Academy of Sciences, Central Lab. of Mechatronics and Instrumentation, BU-1113 Sofia, Bulgaria

${ }^{\text {as }}$ Center for High Energy Physics, Korea Adv. Inst. of Sciences and Technology, 305-701 Taejon, South Korea

${ }^{\text {at }}$ University of Alabama, Tuscaloosa, AL 35486, USA

au Utrecht University and NIKHEF, NL-3584 CB Utrecht, The Netherlands

av Purdue University, West Lafayette, IN 47907, USA

${ }^{\text {aw }}$ Paul Scherrer Institut, PSI, CH-5232 Villigen, Switzerland

${ }^{\text {ax }}$ DESY-Institut fir Hochenergiephysik, D-15738 Zeuthen, FRG

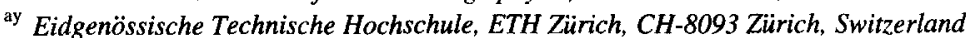

${ }^{\text {az }}$ University of Hamburg, D-22761 Hamburg, FRG

ba High Energy Physics Group, Taiwan, ROC

Received 27 July 1997

Editor: K. Winter

\begin{abstract}
We report on measurements of mass and total decay width of the $\mathrm{W}$ boson and of triple-gauge-boson couplings, $\gamma \mathrm{WW}$ and $\mathrm{ZWW}$, with the $\mathrm{L} 3$ detector at LEP. W-pair events produced in $\mathrm{e}^{+} \mathrm{e}^{-}$interactions between $161 \mathrm{GeV}$ and $172 \mathrm{GeV}$ centre-of-mass energy are selected in a data sample corresponding to a total luminosity of $21.2 \mathrm{pb}^{-1}$. The mass and total decay width of the $\mathrm{W}$ boson are determined to be $M_{\mathrm{W}}=80.75_{-0.27}^{+0.26}\left(\mathrm{exp}\right.$.) \pm 0.03 (LEP) $\mathrm{GeV}$ and $\Gamma_{\mathrm{W}}=1.74_{-0.78}^{+0.88}(\mathrm{stat}$.) \pm 0.25 (syst.) $\mathrm{GeV}$, respectively. Limits on anomalous triple-gauge-boson couplings, $\gamma \mathrm{WW}$ and $\mathrm{ZWW}$, are determined, in particular $-1.5<\delta_{Z}<1.9(95 \% \mathrm{CL})$, excluding vanishing ZWW coupling at more than $95 \%$ confidence level. (C) 1997 Elsevier Science B.V.
\end{abstract}

\section{Introduction}

For the 1996 data taking period, the centre-of-mass energy, $\sqrt{s}$, of the $\mathrm{e}^{+} \mathrm{e}^{-}$collider LEP at CERN was

\footnotetext{
${ }^{1}$ Also supported by CONICET and Universidad Nacional de La Plata, CC 67, 1900 La Plata, Argentina.

${ }^{2}$ Also supported by Panjab University, Chandigarh-160014, India.

${ }^{3}$ Supported by the German Bundesministerium für Bildung, Wissenschaft, Forschung und Technologie.
}

increased to $161 \mathrm{GeV}, 170 \mathrm{GeV}$ and $172 \mathrm{GeV}$. This allowed for the first time the pair-production of on-shell $\mathrm{W}^{ \pm}$bosons in $\mathrm{e}^{+} \mathrm{e}^{-}$interactions, $\mathrm{e}^{+} \mathrm{e}^{-} \rightarrow$ $\mathrm{W}^{+} \mathrm{W}^{-}$. Analysis of $\mathrm{W}$-pair production adds impor-

\footnotetext{
${ }^{4}$ Supported by the National Natural Science Foundation of China.

${ }^{5}$ Supported by the Hungarian OTKA fund under contract numbers $\mathrm{T} 14459$ and $\mathrm{T} 24011$.

${ }^{6}$ Supported also by the Comision Interministerial de Ciencia y Technología.
} 
tant knowledge to the Standard Model of electroweak interactions [1] through the measurements of mass and width of the $\mathrm{W}$ boson and of the triplegauge-boson couplings $\gamma \mathrm{WW}$ and ZWW [2,3]. These parameters were first measured at $\mathrm{p} \overline{\mathrm{p}}$ colliders [4-6].

The total $\mathrm{W}$-pair production cross section as calculated within the Standard Model depends on $\sqrt{s}$ and on the mass and total width of the $\mathrm{W}$ boson, $M_{\mathrm{W}}$ and $\Gamma_{\mathrm{w}}$. Results for $M_{\mathrm{W}}$ derived from total cross section measurements have been published by L3 $[7,8]$ and the other LEP experiments $[9,10]$. In this letter a more precise determination of $M_{\mathrm{W}}$ and a first determination of $\Gamma_{\mathrm{W}}$ is presented based on the invariant mass of the $\mathrm{W}$-boson decay products.

To lowest order within the Standard Model, three Feynman diagrams contribute to $\mathrm{W}$-pair production, the $s$-channel $\gamma$ and $Z$-boson exchange and the $t$-channel $\nu_{\mathrm{e}}$ exchange. The $s$-channel diagrams arise as a consequence of the triple-gauge-boson vertices $\gamma \mathrm{WW}$ and ZWW which are expected due to the non-Abelian gauge structure of the electroweak theory $[1,3]$. Results for triple-gauge-boson couplings derived from the data collected at $\sqrt{s}=161 \mathrm{GeV}$ have been published by L3 $[7,11]$ and the other LEP experiments $[10,12]$. Here a determination of triplegauge-boson couplings is presented based on total and differential cross sections in W-pair mediated four-fermion production.

The L3 detector is described in detail in Ref. [13]. During the 1996 run the L3 detector collected total integrated luminosities of $10.9 \mathrm{pb}^{-1}$ at $\sqrt{\mathrm{s}}=$ 161.34 GeV (threshold data), and $1.0 \mathrm{pb}^{-1}$ and 9.3 $\mathrm{pb}^{-1}$ at $\sqrt{\mathrm{s}}=170.31 \mathrm{GeV}$ and at $\sqrt{s}=172.32 \mathrm{GeV}$ (high-energy data). These centre-of-mass energies are known to $\pm 0.06 \mathrm{GeV}$ [14]. The results obtained at threshold and from the high-energy data are combined to determine the mass of the $\mathrm{W}$ boson and triple-gauge-boson couplings.

\section{Analysis of four-fermion production}

The $\mathrm{W}$ boson decays into a quark-antiquark pair, such as $\mathrm{W}^{-} \rightarrow \overline{\mathrm{u}} \mathrm{d}$ or $\overline{\mathrm{cs}}$, or a lepton-antilepton pair, $\mathrm{W}^{-} \rightarrow \ell^{-} \bar{v}_{\ell}(\ell=\mathrm{e}, \mu, \tau)$; in the following denoted as $q q, \ell \nu$ or $f f$ in general for both $\mathrm{W}^{+}$and $\mathrm{W}$ decays. All four-fermion final states expected in W-pair production are analysed:
1. $\mathrm{e}^{+} \mathrm{e}^{-} \rightarrow \mathrm{qqe} v(\gamma)$,

2. $\mathrm{e}^{+} \mathrm{e}^{-} \rightarrow \mathrm{qq} \mu \nu(\gamma)$,

3. $\mathrm{e}^{+} \mathrm{e}^{-} \rightarrow \mathrm{qq} \tau \nu(\gamma)$,

4. $\mathrm{e}^{+} \mathrm{e}^{-} \rightarrow \ell v \ell \nu(\gamma)$

5. $\mathrm{e}^{+} \mathrm{e}^{-} \rightarrow \mathrm{qqqq}(\gamma)$,

where $(\gamma)$ indicates the possible presence of radiative photons. The selections of these five four-fermion final states are described in detail in Ref. [7] for the threshold data and in Ref. [8] for the high-energy data.

These analyses reconstruct the visible fermions in the final state, i.e., electrons, muons, $\tau$ jets corresponding to the visible $\tau$ decay products, and hadronic jets corresponding to quarks $[7,8]$. Kinematic constraints as discussed below are then imposed to improve the resolution in the measured fermion energies and angles and to determine those not measured.

Parameters such as the mass or width of the W boson or triple-gauge-boson couplings are determined by comparing samples of Monte Carlo events to the data. A reweighting procedure is applied to construct Monte Carlo samples with different parameters. Selection, resolution and other detector effects are determined locally in phase space by averaging over Monte Carlo events inside a multi-dimensional box around each data event.

The following Monte Carlo event generators are used to simulate the various signal and background reactions: KORALW [15] and HERWIG [16] $\left(\mathrm{e}^{+} \mathrm{e}^{-}\right.$ $\rightarrow \mathrm{WW} \rightarrow \operatorname{fff}(\gamma))$; EXCALIBUR [17] $\left(\mathrm{e}^{+} \mathrm{e}^{-} \rightarrow\right.$ $f f f f(\gamma)) ;$ PYTHIA [18] $\left(\mathrm{e}^{+} \mathrm{e}^{-} \rightarrow q \bar{q}(\gamma), \mathrm{ZZ}(\gamma)\right.$, hadronic two-photon collisions); KORALZ [19] $\left(\mathrm{e}^{+} \mathrm{e}^{-} \rightarrow \mu^{+} \mu^{-}(\gamma), \tau^{+} \tau^{-}(\gamma)\right)$; BHAGENE3 [20] $\left(\mathrm{e}^{+} \mathrm{e}^{-} \rightarrow \mathrm{e}^{+} \mathrm{e}^{-}(\gamma)\right)$. The response of the $\mathrm{L} 3$ detector is modelled with the GEANT [21] detector simulation program which includes the effects of energy loss, multiple scattering and showering in the detector materials and in the beam pipe.

\subsection{Event reconstruction imposing kinematic con- straints}

The final states $q q \mathrm{e} v, q q \mu \nu$ and $q q q q$ contain at most one unmeasured neutrino, so a kinematic fit is applicable. The kinematic fit determines energy, $E_{f}$, polar angle, $\theta_{f}$, and azimuthal angle, $\phi_{f}$, for all four fermions, $f$, in the final state. It adjusts the measure- 
ments of these quantities for the visible fermions according to their experimental resolutions to satisfy the constraints imposed. For hadronic jets, the velocity $\beta_{f}=\left|p_{f}\right| / E_{f}$ of the jet is kept at its measured value as systematic effects cancel in the ratio. Fourmomentum conservation and equal mass of the two W bosons are imposed as constraints. They allow the determination of the unmeasured neutrino momentum vector. For $q q \mathrm{e} \nu$ and $q q \mu \nu$ events, this yields a $2 \mathrm{C}$ kinematic fit, whereas for $q q q q$ events it is a $5 \mathrm{C}$ kinematic fit.

The kinematic fit mainly improves the energy resolution and less the angular resolutions. The resolutions in average invariant mass, $M_{\text {inv }}$, typically improve by a factor of three.

For $q q \tau \nu$ and $\ell \nu \ell \nu$ events, the event contains at least two unmeasured neutrinos in the final state. In case of $q q \tau \nu$ events, the energies of the two hadronic jets are rescaled by a common factor so that their sum equals half the centre-of-mass energy. The $\tau$ direction of flight is approximated by the direction of the visible $\tau$ jet. The $\tau$ energy and the neutrino momentum vector are then determined by overall energy-momentum conservation. This yields two equal-mass $\mathrm{W}$ bosons. The $\ell \nu \ell \nu$ events are used in the determination of triple-gauge-boson couplings only.

\subsection{Fitting method for mass, width and gauge cou- plings}

The maximum likelihood method is used to extract values and errors of parameters, $\Psi$, such as the mass and total width of the $\mathrm{W}$ boson or triplegauge-boson couplings. The fit considers a set of values of reconstructed quantities $\Omega$ for each data event, which are either the average invariant mass, $M_{\text {inv }}$, or phase-space angles describing the four-fermion final state (see Section 2.4 below). The data are treated as unbinned; the total likelihood is the product of the normalised differential cross section, $L(\Omega, \Psi)$, for all data events. For a given four-fermion final state $i$, one has:

$$
\begin{aligned}
L_{i}\left(\Omega_{i}, \Psi\right)= & \frac{1}{\sigma_{i}(\Psi)+\sigma_{i}^{\mathrm{BG}}} \\
& \times\left[\frac{\mathrm{d} \sigma_{i}\left(\Omega_{i}, \Psi\right)}{\mathrm{d} \Omega_{i}}+\frac{\mathrm{d} \sigma_{i}^{\mathrm{BG}}\left(\Omega_{i}\right)}{\mathrm{d} \Omega_{i}}\right],
\end{aligned}
$$

where $\sigma_{i}$ and $\sigma_{i}^{\mathrm{BG}}$ are the accepted signal and background cross sections. The total and differential cross sections of the accepted background are independent of the parameters $\Psi$ of interest. They are taken directly from Monte Carlo simulations.

The total and differential signal cross sections depend on $\Psi$. For values $\Psi_{\text {fit }}$ varied during the fitting procedure, they are determined by a reweighting procedure applied to Monte Carlo events originally generated with parameter values $\Psi_{\text {gen }}$. For mass and width fits, the event weights $R_{i}$ are given by the ratio:

$$
\begin{aligned}
& R_{i}\left(m_{1}, m_{2}, \Psi_{\text {fit }}, \Psi_{\text {gen }}\right) \\
& \quad=\frac{\mathrm{d}^{2} \sigma_{i}\left(s, m_{1}, m_{2}, \Psi_{\text {fit }}\right) / \mathrm{d} m_{1} \mathrm{~d} m_{2}}{\mathrm{~d}^{2} \sigma_{i}\left(s, m_{1}, m_{2}, \Psi_{\text {gen }}\right) / \mathrm{d} m_{1} \mathrm{~d} m_{2}}
\end{aligned}
$$

where $m_{1}$ and $m_{2}$ are the invariant masses of the two generated $\mathrm{W}$ bosons. The differential cross sections are calculated with the GENTLE [22] program. For couplings fits, the event weights $R_{i}$ are calculated as the ratio:

$$
\begin{aligned}
R_{i} & \left(p_{1}, p_{2}, p_{3}, p_{4}, k_{\gamma}, \Psi_{\text {fit }}, \Psi_{\text {gen }}\right) \\
& =\frac{\left|\mathscr{M}_{i}\left(p_{1}, p_{2}, p_{3}, p_{4}, k_{\gamma}, \Psi_{\text {fit }}\right)\right|^{2}}{\left|\mathscr{M}_{i}\left(p_{1}, p_{2}, p_{3}, p_{4}, k_{\gamma}, \Psi_{\mathrm{gen}}\right)\right|^{2}},
\end{aligned}
$$

where $\mathscr{K}_{i}$ is the matrix element of the four-fermion final state $i$ under consideration evaluated for the generated four-vectors $\left(p_{1}, p_{2}, p_{3}, p_{4}, k_{\gamma}\right)$ of the four fermions and any radiative photons. The matrix elements as implemented in the EXCALIBUR [17] event generator are used, which include all relevant tree-level Feynman diagrams contributing to a given four-fermion final state. The total accepted signal cross section for a given set of parameters $\Psi_{\text {fit }}$ is then:

$\sigma_{i}\left(\Psi_{\text {fit }}\right)=\frac{\sigma_{i}^{\text {gen }}}{N_{i}^{\text {gen }}} \cdot \sum_{j} R_{i}\left(j, \Psi_{\text {fit }}, \Psi_{\text {gen }}\right)$,

where $\sigma_{i}^{\text {gen }}$ denotes the cross section corresponding to the total Monte Carlo sample containing $N_{i}^{\text {gen }}$ events. The sum extends over all accepted Monte Carlo events $j$. The accepted differential signal cross section in reconstructed quantities $\Omega_{i}$ is determined 
by averaging Monte Carlo events inside a box in $\Omega_{i}$ around each data event [23]:

$$
\frac{\mathrm{d} \sigma_{i}\left(\Omega_{i}, \Psi_{\mathrm{fit}}\right)}{\mathrm{d} \Omega_{i}}=\frac{\sigma_{i}^{\mathrm{gen}}}{N_{i}^{\mathrm{gen}}} \cdot \frac{1}{\Delta_{i}^{\Omega}} \sum_{j \in \Delta_{i}^{\Omega}} R_{i}\left(j, \Psi_{\mathrm{fit}}, \Psi_{\mathrm{gen}}\right),
$$

where $\Delta_{i}^{\Omega}$ is the volume of the box and the sum extends over all accepted Monte Carlo events $j$ inside the box. This takes $\Omega_{i}$-dependent detector effects and $\Psi$-dependent efficiencies and purities properly into account.

In addition, extended maximum likelihood fits are performed by including the overall normalisations according to the measured total W-pair cross sections. The likelihood is multiplied by the Poissonian probabilities to obtain the numbers of events observed in the data $[7,8]$ given the integrated luminosities and the expectations for the total accepted signal and background cross sections, $\sigma_{i}\left(\Psi_{\text {fit }}\right)$ and $\sigma_{i}{ }^{\mathrm{BG}}$, at all centre-of-mass energies.

The fit method described above determines the parameters without any bias as long as the Monte Carlo describes photon radiation (ISR) and detector effects such as resolution and acceptance functions correctly. By fitting large Monte Carlo samples, typically a hundred times the data, the fitting procedure is tested to high accuracy. The fits reproduce well the values of the parameters of the large Monte Carlo samples being fitted. Also, the fit results do not depend on the values of the parameters $\Psi_{\text {gen }}$ of the Monte Carlo sample subjected to the reweighting procedure.

\subsection{Mass and width of the $W$ boson}

For mass and width fits, the weighted average of the two invariant masses in an event, $M_{\text {inv }}$, as determined by the kinematic fit imposing the equal-mass constraint, is fitted. The size of the box around each data event is limited by the requirement of including no more than 1000 Monte Carlo events, yielding box sizes of about $\pm 35 \mathrm{MeV}$ at the peak of the invariant mass distribution. In addition, the box size may not be larger than $\pm 250 \mathrm{MeV}$ around $M_{\mathrm{inv}}$.

Based on the high-energy data, the mass of the $\mathrm{W}$ boson is determined for each of the final states $q q \mathrm{e} v$ (19 events), $q q \mu \nu$ ( 9 events), $q q \tau \nu$ (12 events) and qqqq (61 events) in separate maximum likelihood fits. Combined results are determined by multiplying the likelihood of the individual channels. For mass fits in the $q q q q$ channel, the pairing algorithm to assign jets to $\mathrm{W}$ bosons used in the event selection [8] is changed. The pairing yielding the highest likelihood in the $5 \mathrm{C}$ kinematic fit is chosen. The rate of correct pairings is reduced to $60 \%$ for the best combination and it is $25 \%$ for the second best combination. However, the signal-to-background ratio in the relevant signal region around $M_{\mathrm{inv}} \approx 80 \mathrm{GeV}$ is improved. The loss of correct pairings is recovered by including the pairing with the second highest likelihood in the fits. Monte Carlo studies show that the two values for $M_{\mathrm{w}}$ obtained from fitting the distributions of the best and the second best combination separately have a correlation of $(1.4 \pm 2.2) \%$, which is negligible.

The observed invariant mass distributions together with the fit results are shown in Figs. 1 and 2. The results on $M_{\mathrm{w}}$ are summarised in Table 1. The observed statistical errors agree well with the statistical errors expected for the size of the high-energy data samples used. Systematic errors on the fitted W masses are summarised in Table 2. Hadronisation and fragmentation effects are determined by comparing different Monte Carlo programs to simulate the signal. Effects due to background are determined by varying both the total accepted background cross section and the shape of the invariant mass spectrum. Detector effects due to uncertainties in the energy scale of electrons, muons and hadronic jets and the corresponding resolutions are estimated by varying

Table 1

Results on the mass of the $\mathrm{W}$ boson, $M_{\mathrm{W}}$, for the individual four-fermion final states in W-pair production, and their combination. The first error is statistical and the second systematic

\begin{tabular}{cc}
\hline Process & $\begin{array}{c}\text { Mass of the W boson } M_{\mathrm{W}} \\
{[\mathrm{GeV}]}\end{array}$ \\
\hline $\mathrm{e}^{+} \mathrm{e}^{-} \rightarrow \mathrm{qqe} \nu(\gamma)$ & $80.25_{-0.70}^{+0.68} \pm 0.09$ \\
$\mathrm{e}^{+} \mathrm{e}^{-} \rightarrow \mathrm{qq} \mu \nu(\gamma)$ & $80.94_{-1.33}^{+1.15} \pm 0.08$ \\
$\mathrm{e}^{+} \mathrm{e}^{-} \rightarrow \mathrm{qq} \tau \nu(\gamma)$ & $80.43_{-1.06}^{+1.07} \pm 0.09$ \\
$\mathrm{e}^{+} \mathrm{e}^{-} \rightarrow q q \ell \nu(\gamma)$ & $80.42_{-0.55}^{+0.53} \pm 0.07$ \\
$\mathrm{e}^{+} \mathrm{e}^{-} \rightarrow \mathrm{qqqq}(\gamma)$ & $80.91_{-0.44}^{+0.41} \pm 0.13$ \\
$\mathrm{e}^{+} \mathrm{e}^{-} \rightarrow$ ffff $(\gamma)$ & $80.71_{-0.35}^{+0.34} \pm 0.09$ \\
\hline
\end{tabular}



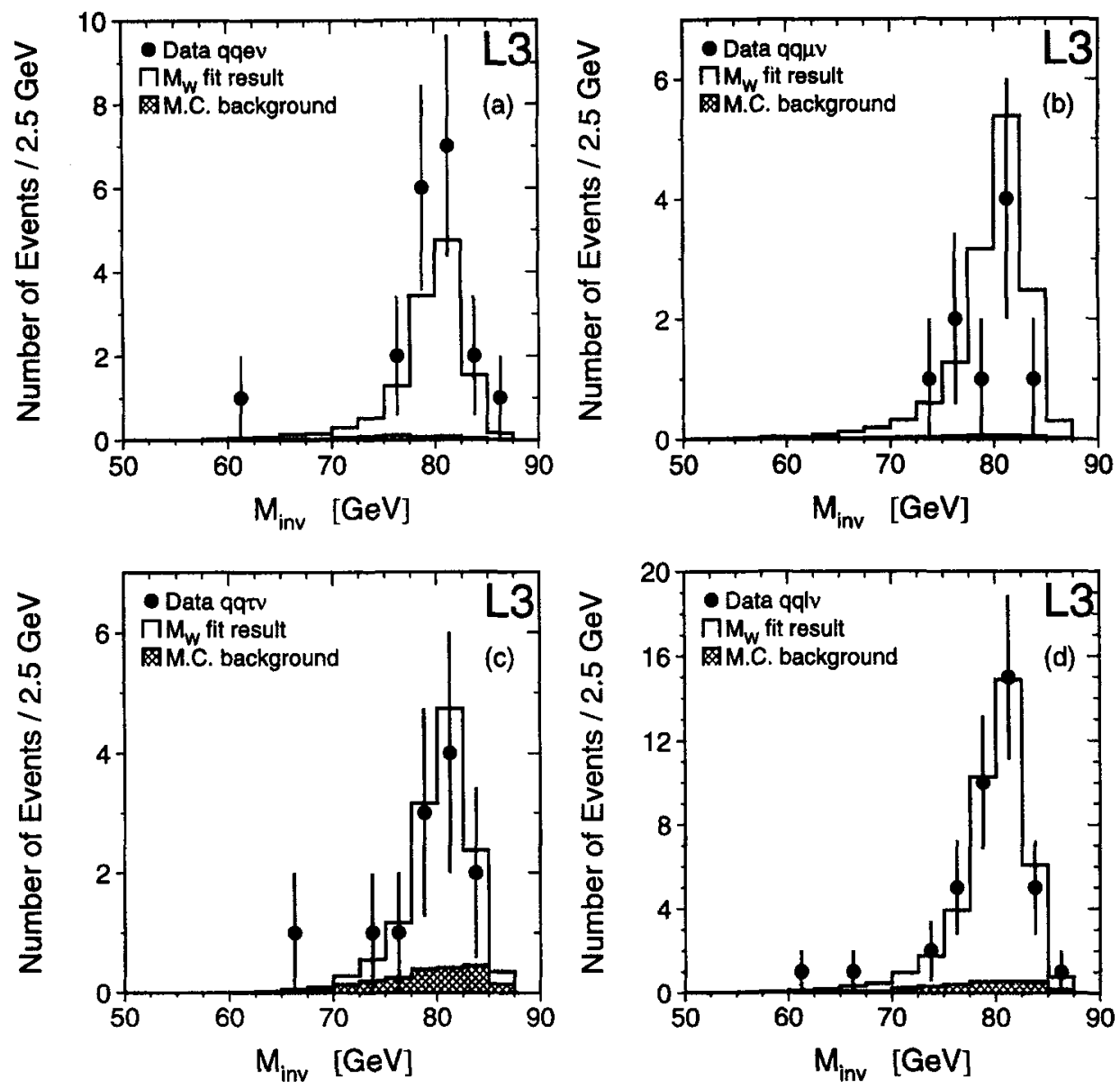

Fig. 1. Distributions of reconstructed invariant mass after applying the kinematic fit using the equal-mass constraint, $M_{\text {inv }}$, for selected events in the channels (a) $q q \mathrm{e} \nu$, (b) $q q \mu \nu$, (c) $q q \tau \nu$, (d) $q q \ell \nu$, combining $q q e \nu, q q \mu \nu$ and $q q \tau \nu$. The solid lines show the result of the fits of $M_{\mathrm{W}}$ to the indicated final states.

them within their errors. The systematic error due to the fitting method includes effects due to different reweighting procedures and technical parameters such as box size and occupancy. For each individual channel and their combinations the total systematic error is small compared to the statistical error.

The results on $M_{\mathrm{W}}$ determined in the $q q \mathrm{e} v$, $q q \mu \nu$, and $q q \tau \nu$ final states are in good agreement with each other. They are averaged in a combined fit and compared to the result on $M_{\mathrm{w}}$ determined in the $q q q q$ final state:

$M_{\mathrm{W}}(q q \ell \nu)=80.42_{-0.55}^{+0.52}$ (stat.) \pm 0.07 (syst.) $\mathrm{GeV}$ $M_{\mathrm{W}}(q q q q)=80.91_{-0.44}^{+0.41}$ (stat.) \pm 0.13 (syst.) $\mathrm{GeV}$. Within the statistical accuracy of these measurements there is no difference between $M_{\mathrm{W}}$ as deter- mined in $q q \ell \nu$ and $q q q q$ events. Differences may arise due to possible strong final-state interactions (FSI) in $q q q q$ events, such as colour-reconnection (CR) [24] or Bose-Einstein (BE) [25] effects. Depending on the details of the Monte Carlo modelling [26,2], mass shifts of up to $100 \mathrm{MeV}$ are possible, which are small compared to the current statistical error and accounted for in the systematic error. Averaging the two results on $M_{\mathrm{w}}$ in a combined fit yields:

$M_{\mathrm{W}}=80.71_{-0.35}^{+0.34}$ (stat.) \pm 0.09 (syst.) GeV.

The observed mass distribution is shown in Fig. 3a and compared to the expectation based on this $\mathrm{W}$ mass value. In order to determine also the total decay 

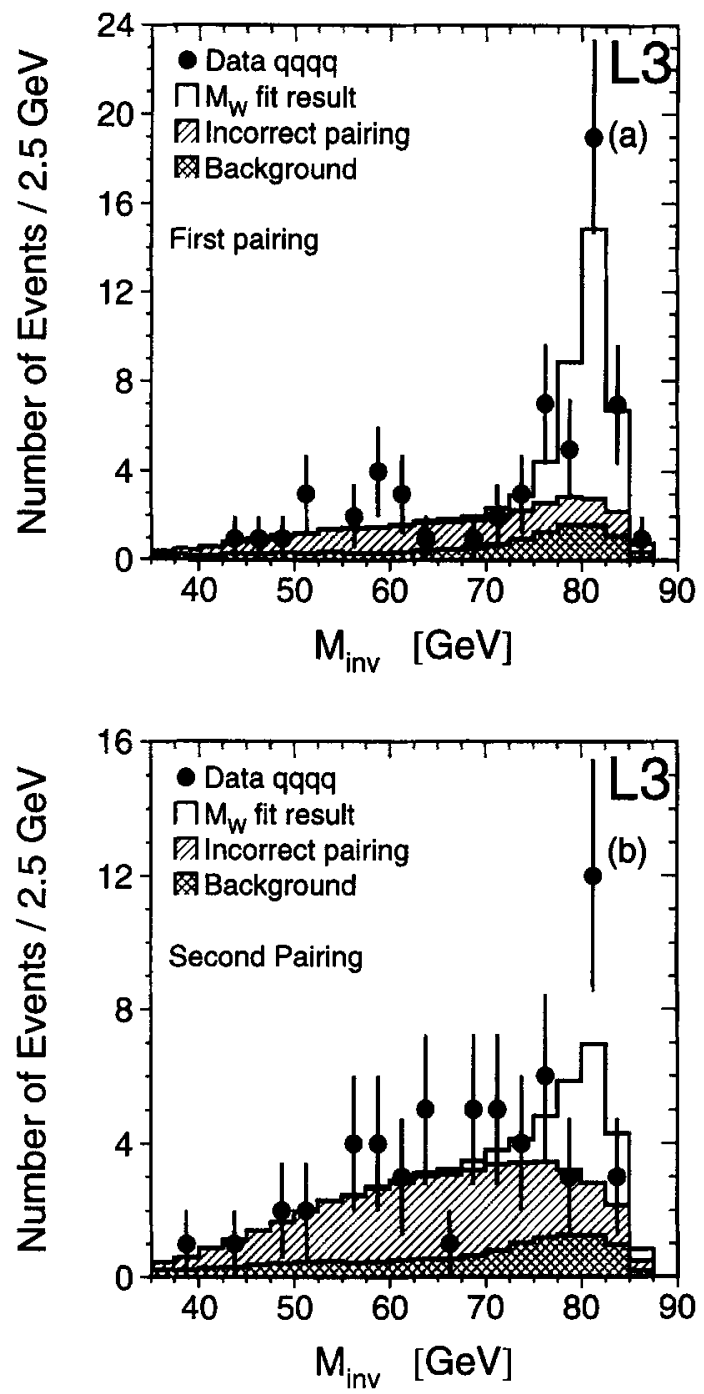

Fig. 2. Distributions of reconstructed invariant mass after applying the kinematic fit using the equal-mass constraint, $M_{\text {inv }}$, for selected events in the channel qqqq: (a) first pairing, i.e., pairing with highest $5 \mathrm{C}$ likelihood, (b) second pairing, i.e., pairing with second highest $5 \mathrm{C}$ likelihood. The solid lines show the result of the fit of $M_{\mathrm{W}}$ to both $q q q q$ pairings.

width of the $\mathrm{W}$ boson, $\Gamma_{\mathrm{W}}$ is treated as an independent parameter instead of imposing the Standard Model calculation $\Gamma_{\mathrm{w}}=\Gamma_{\mathrm{w}}\left(M_{\mathrm{w}}\right)$ [26]. The results are listed in Table 3 . The mass values obtained in these fits are nearly the same as before. The mass errors differ according to the width fitted being larger or smaller than the width expected in the
Standard Model. For all final states combined the result is:

$M_{\mathrm{W}}=80.72_{-0.33}^{+0.31}$ (stat.) \pm 0.09 (syst.) GeV

$\Gamma_{\mathrm{W}}=1.74_{-0.78}^{+0.88}$ (stat.) \pm 0.25 (syst.) GeV,

with a correlation coefficient of $+27 \%$ between $M_{\mathrm{W}}$ and $\Gamma_{\mathrm{w}}$. The result of this fit is compared to the data in Fig. 3b. Systematic errors on the fitted $W$ widths are summarised in Table 4. Our result on $\Gamma_{\mathrm{w}}$ is in good agreement with the measurement at $\mathrm{p} \overline{\mathrm{p}}$ colliders, $2.07 \pm 0.06 \mathrm{GeV}$ [5]. It also agrees well with the Standard Model expectation, $2.08 \mathrm{GeV}$ [26].

As cross checks, other methods to extract a value for the W-boson mass from the distribution of the average reconstructed invariant mass are studied, in particular the methods referred to as Monte Carlo calibration method and convolution method [2]. The Monte Carlo calibration method uses a simple function to describe the observed invariant mass distribution. One of the fit parameters is used as an estimator for the W-boson mass which is calibrated by fitting samples of Monte Carlo events with known W masses. The Monte Carlo convolution method uses the theoretically expected average invariant mass distribution convoluted with the detector resolution. Within the errors, the same results are obtained with either method.

The results on $M_{\mathrm{W}}$ presented here agree very

Table 2

Systematic errors in the determination of $M_{\mathrm{W}}$ for the different final states $[\mathrm{MeV}]$. The contributions listed in the upper part are treated as correlated when combining different final states. The other contributions are treated as uncorrelated between channels

\begin{tabular}{ccccc}
\hline Source & \multicolumn{4}{c}{ Final state } \\
\cline { 2 - 5 } & $q q \mathrm{e} \nu$ & $q q \mu \nu$ & $q q \tau \nu$ & $q q q q$ \\
\hline$\sqrt{s}$ & 30 & 30 & 30 & 30 \\
ISR & 10 & 10 & 10 & 10 \\
Hadronisation & 40 & 40 & 40 & 40 \\
Fitting Method & 55 & 30 & 30 & 30 \\
FSI (CR + BE) & -- & -- & -- & 100 \\
Background & 25 & 15 & 50 & 15 \\
Energy Scales & 30 & 20 & -- & 10 \\
Resolutions & 5 & 5 & 10 & 45 \\
Monte Carlo Statistics & 40 & 40 & 40 & 40 \\
& & & & \\
Total & 90 & 80 & 90 & 130 \\
\hline
\end{tabular}


Table 3

Results on the mass of the $\mathrm{W}$ boson, $M_{\mathrm{W}}$, its total decay width, $\Gamma_{\mathrm{W}}$, and their correlation

\begin{tabular}{cccc}
\hline Process & $\begin{array}{c}\text { Mass of the W boson } M_{\mathrm{W}} \\
{[\mathrm{GeV}]}\end{array}$ & $\begin{array}{c}\text { Total decay width } \Gamma_{\mathrm{w}} \\
{[\mathrm{GeV}]}\end{array}$ & $\begin{array}{c}\text { Correlation } \\
\text { coefficient }\end{array}$ \\
\hline $\mathrm{e}^{+} \mathrm{e}^{-} \rightarrow q q \ell \nu(\gamma)$ & $80.43_{-0.58}^{+0.64} \pm 0.07$ & $2.76_{-1.46}^{+1.93} \pm 0.28$ & +0.33 \\
$\mathrm{e}^{+} \mathrm{e}^{-} \rightarrow \mathrm{qqqq}(\gamma)$ & $80.94_{-0.36}^{+0.35} \pm 0.13$ & $1.21_{-1.20}^{+0.77} \pm 0.33$ & +0.11 \\
$\mathrm{e}^{+} \mathrm{e}^{-} \rightarrow$ ffff $(\gamma)$ & $80.72_{-0.33}^{+0.31} \pm 0.09$ & $1.74_{-0.78}^{+0.88} \pm 0.25$ & +0.27 \\
\hline
\end{tabular}
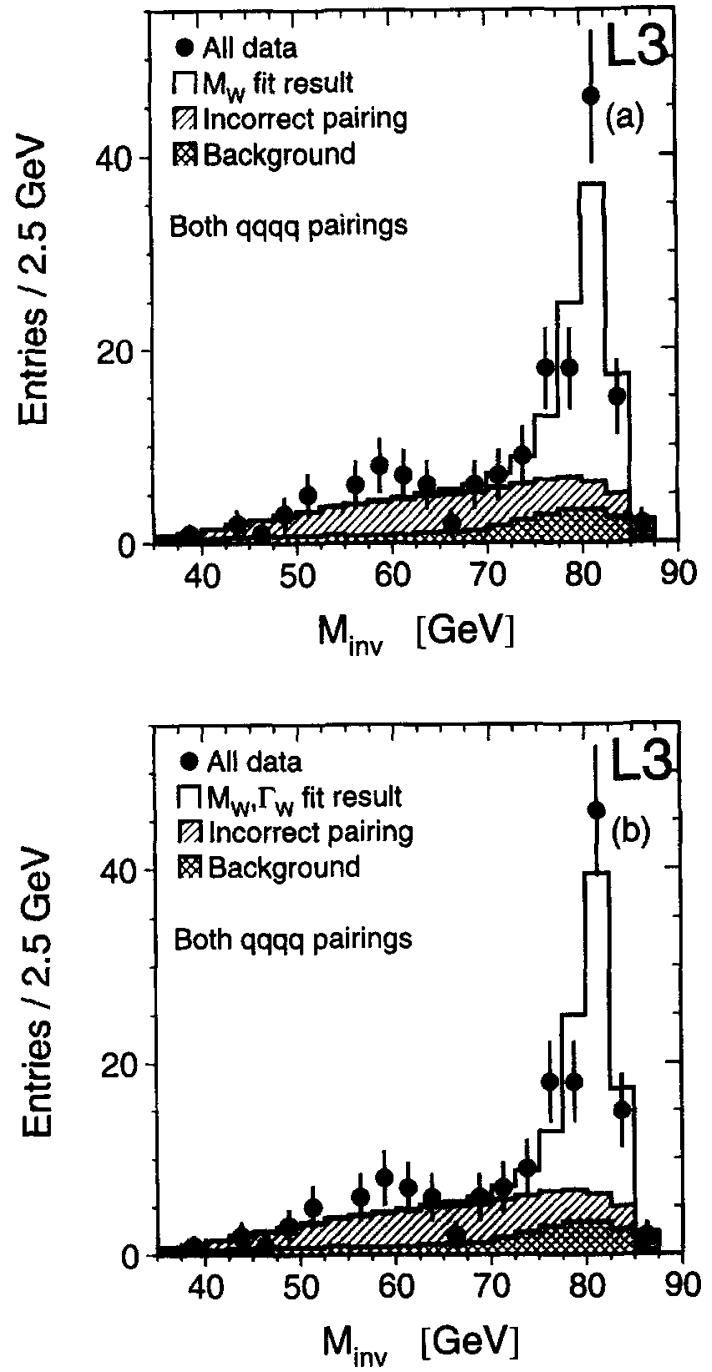

Fig. 3. Distribution of reconstructed invariant mass after applying the kinematic fit using the equal-mass constraint, $M_{\text {inv }}$, for all selected events, entering both first and second pairing in the $q q q q$ channel. The solid line shows the result of the fit of (a) $M_{\mathrm{W}}$ and (b) $M_{\mathrm{W}}$ and $\Gamma_{\mathrm{w}}$ to all data. well with our result derived from the measurements of the total W-pair production cross section, $M_{\mathrm{W}}=$ $80.78_{-0.41}^{+0.45}$ (exp.) \pm 0.03 (LEP) GeV [8]. Combining both results in an extended maximum likelihood fit yields:

$M_{\mathrm{W}}=80.75_{-0.27}^{+0.26}$ (exp.) \pm 0.03 (LEP) GeV.

This direct determination of $M_{\mathrm{W}}$ is in agreement with the direct determination of $M_{\mathrm{W}}$ at $\mathrm{p} \overline{\mathrm{p}}$ colliders, $80.33 \pm 0.15 \mathrm{GeV}$ [4]. It also agrees with our indirect determination of $M_{\mathrm{W}}$ through radiative corrections measured at the $\mathrm{Z}$ peak, $M_{\mathrm{W}}=80.22 \pm 0.22 \mathrm{GeV}$ [27], testing the Standard Model at the level of its electroweak corrections.

\subsection{Gauge couplings of the $W$ boson}

Anomalous contributions to the triple-gauge-boson vertices $\gamma \mathrm{WW}$ and $\mathrm{ZWW}$ are parametrised in terms of seven complex triple-gauge-boson couplings each [28], too many to be measured simultaneously. Therefore, models are considered which re-

Table 4

Systematic errors in the determination of $\Gamma_{\mathrm{W}}$ for the different final states $[\mathrm{MeV}]$. The error arising due to the fitting method is treated as correlated when combining different final states. The other contributions are treated as uncorrelated between channels

\begin{tabular}{ccc}
\hline Source & \multicolumn{2}{c}{ Final state } \\
\cline { 2 - 3 } & $q q \ell \nu$ & $q 9 q 9$ \\
\hline Fitting method & 200 & 200 \\
Background & 90 & 200 \\
Energy scales & 50 & 50 \\
Resolutions & 150 & 150 \\
Monte Carlo statistics & 60 & 60 \\
Total & 280 & 330 \\
\hline
\end{tabular}


duce the number of free parameters to one or two by making additional assumptions [29-31,3].

First it is interesting to test if the coupling between the $\mathrm{Z}$ and a pair of $\mathrm{W}$ bosons exists [31]. Neglecting the contributions of dimension-six operators, assuming that all electromagnetic properties of the $\mathrm{W}$ boson are standard and that a custodial SU(2) symmetry is respected leaves a single parameter, $\delta_{Z}$ [31]. This parameter describes the deviation of the ZWW coupling, gZWW, from its Standard Model value of $\cot \theta_{\mathrm{W}} \approx 1.9$, where $\theta_{\mathrm{W}}$ is the electroweak mixing angle. The model is extended to include anomalous electromagnetic properties of the $\mathrm{W}$ boson, such as an additional contribution to its magnetic moment, $\Delta \kappa_{\gamma}=\kappa_{\gamma}-1$ [31].

Other models consider only CP-conserving dimension-six operators neither affecting the gaugeboson propagators at tree level nor generating anomalous Higgs couplings [3]. In that case there are three operators giving rise to deviations in the $\mathrm{C}$ - and P-conserving triple-gauge-boson couplings, with corresponding anomalous couplings denoted as $\alpha_{\mathrm{W} \Phi}$, $\alpha_{\mathrm{W}}$ and $\alpha_{\mathrm{B} \phi}$ [3].

In case of triple-gauge-boson couplings fits, a
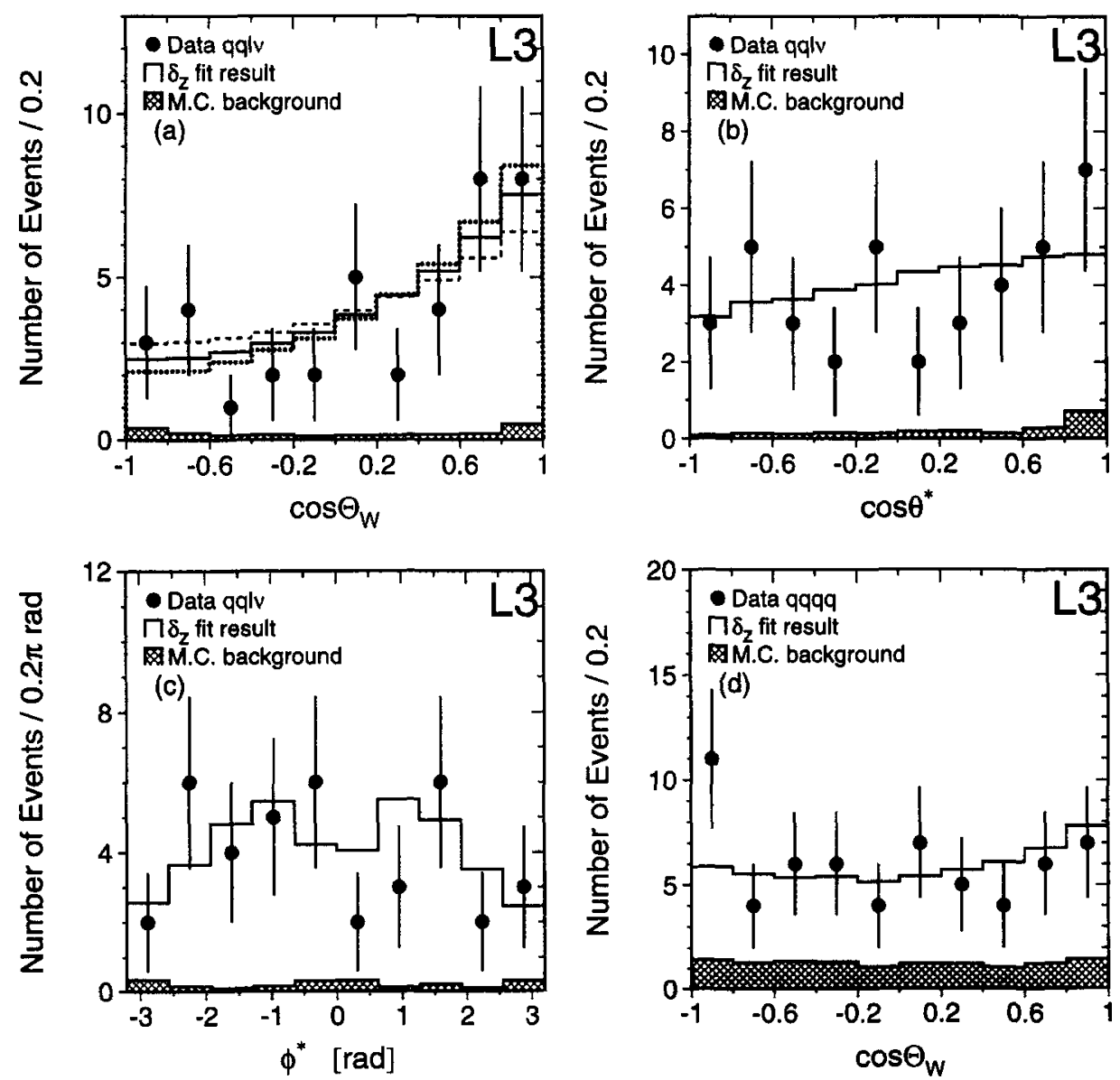

Fig. 4. Distributions of reconstructed phase-space angles after applying the kinematic fit using the equal-mass constraint. The solid lines show the result of the $\delta_{\mathrm{Z}}$ fit to the data. (a) The polar angle of the $\mathrm{W}^{-}$boson, $\cos \Theta_{\mathrm{w}}$, for selected $q q \ell \nu$ events. The dotted and dashed lines correspond to the positive and negative $68 \% \mathrm{CL}$ errors on the fitted $\delta_{Z}$. (b) The polar decay angle of the leptonically decaying W boson, $\cos \theta^{*}$, for selected $q q \ell \nu$ events. (c) The azimuthal decay angle of the leptonically decaying W boson, $\phi^{*}$, for selected $q q \ell \nu$ events. The value of $\phi^{*}$ is shifted by $\pi$ for $W^{-}$decays in order to have the same $\phi^{*}$ distribution for $W^{-}$and $W^{+}$decays. (d) The polar angle of the $\mathrm{W}^{-}$boson, $\cos \Theta_{\mathrm{W}}$, for selected $q q q q$ events. 
Table 5

Results on anomalous triple-gauge-boson couplings for $68 \%$ and 95\% confidence level. Results of the four fits to one anomalous coupling are listed in the upper part. In the lower part, results of the fit to two anomalous couplings are listed. The correlation between $\delta_{z}$ and $\Delta \kappa_{\gamma}$ is $-30 \%$. The errors are total errors combining statistical and systematic errors

\begin{tabular}{ccc}
\hline Coupling & \multicolumn{2}{c}{ Result } \\
\cline { 2 - 3 } & $68 \% \mathrm{CL}$ & $95 \% \mathrm{CL}$ \\
\hline$\delta_{\mathrm{Z}}$ & $0.10_{-0.92}^{+1.03}$ & $0.10_{-1.57}^{+1.76}$ \\
$\alpha_{\mathrm{W} \Phi}$ & $0.04_{-0.43}^{+0.43}$ & $0.04_{-0.63}^{+0.81}$ \\
$\alpha_{\mathrm{W}}$ & $0.22_{-0.61}^{+0.59}$ & $0.22_{-1.08}^{+1.04}$ \\
$\alpha_{\mathrm{B} \Phi}$ & $0.07_{-1.16}^{+1.77}$ & $0.07_{-1.94}^{+2.80}$ \\
& & \\
$\delta_{\mathrm{Z}}$ & $0.09_{-0.94}^{+1.05}$ & $0.09_{-1.57}^{+1.92}$ \\
$\Delta \kappa_{\gamma}$ & $0.31_{-1.12}^{+1.81}$ & $0.31_{-1.91}^{+2.96}$ \\
\hline
\end{tabular}

subset of the five phase-space angles describing the four-fermion final state is fitted. Fixing the mass of the $\mathrm{W}$ boson [4] and neglecting photon radiation and final-state fermion helicities, five phase-space angles completely describe the four-fermion final state for unpolarised initial states. These are the polar angle of the $\mathrm{W}^{-}$boson, $\cos \Theta_{\mathrm{w}}$, and the poiar and azimuthai decay angles in the rest systems of the two decaying $W$ bosons, $\cos \theta_{ \pm}^{*}$ and $\phi_{ \pm}^{*}$, for the fermion and antifermion in $\mathrm{W}^{-}$and $\mathrm{W}^{+}$decay, respectively.

For charged leptons, the sign of their electric charge determines whether they are fermions or antifermions. For hadronic jets, the flavour and charge of the original quark is not measured. Thus, there arises a two-fold ambiguity in the decay angles of hadronicaliy decaying $\mathbb{W}$ bosons, $\left(\cos \theta^{*}, \phi^{*}\right) \leftrightarrow$ $\left(-\cos \theta^{*}, \pi+\phi^{*}\right)$. If both $\mathrm{W}$ bosons decay hadronically, the polar angle of the $W^{-}$boson also has a two-fold ambiguity, $\cos \Theta_{\mathrm{W}} \leftrightarrow-\cos \Theta_{\mathrm{W}}$. This ambiguity is resolved by combining the jet charges of each pair of jets to determine the charge of each $W$. The difference in the two $W$ charges is then used to determine the sign of $\cos \Theta_{w}$. The charge of each jet is determined from a rapidity weighted sum of the tracks reconstructed in the central tracking chamber and assigned to the jet with rapidity weight $\kappa=1.0$ [32]. The sign assignment is found to be correct $67 \%$ of the time when the jets are correctly paired.

Only the high-energy data enter the fitted differential distributions. For semileptonic $q q \ell \nu$ events, the variables $\Omega_{i}$ considered in the fit are $\cos \Theta_{\mathrm{W}}$ and the decay angles of the leptonically decaying $W$ boson, $\cos \theta^{*}$ and $\phi^{*}$. A total of $39 q q \ell \nu$ events are used. One $q q \tau \nu$ event is rejected because two tracks with opposite charge are associated with the $\tau$ jet. A total of 60 hadronic $q q q q$ events are used for which $\cos \Theta_{\mathrm{w}}$ is determined. The distributions of these phase-space angles are shown in Fig. 4. The one- and three-dimensional boxes are constructed in such a way that the mean of the Monte Carlo events inside the box coincides with the data event, leading to asymmetric boxes. The box size is increased until at least 350 Monte Carlo events are included unless a phase-space boundary is reached first. Both the threshold data [7] and the high-energy data [8] are used in the total cross section measurements included in the fit for all five final states. For total cross sections, the EXCALIBUR predictions are scaled to match the GENTLE predictions to account for the more complete calculation of radiative corrections by GENTLE.

Results on anomalous contributions to triplegauge-boson couplings based on these extended maximum likelihood fits are summarised in Table 5. The fitted statistical errors agree well with the statistical errors expected for the size of the data sample used. Besides leading to a bias, systematic effects may also change the sensitivity and thus the statistical errors as given by the fit. Both effects are taken into account. Systematic errors on the fitted triplegauge-boson couplings are summarised in Table 6. The total systematic error for all couplings is dominated by the uncertainties in the global selection efficiencies entering the total cross section part of the likelihood. Systematic errors due to uncertainties in $M_{\mathrm{W}}, \sqrt{s}$ and detectôr resolutions are small. Ef-

Table 6

Systematic errors in the determination of triple-gauige-boson coùplings

\begin{tabular}{ccccc}
\hline Source & \multicolumn{5}{c}{ Systematic errors on } \\
\cline { 2 - 6 } & $\delta_{z}$ & $\alpha_{\mathrm{w} \Phi}$ & $\alpha_{\mathrm{W}}$ & $\alpha_{\mathrm{B} \Phi}$ \\
\hline$M_{\mathrm{w}}$ & 0.06 & 0.02 & 0.06 & 0.11 \\
$\sqrt{s}$ & 0.02 & 0.01 & 0.02 & 0.04 \\
Selection efficiencies & 0.41 & 0.16 & 0.22 & 0.62 \\
Resolutions and energy scales & 0.03 & 0.02 & 0.02 & 0.03 \\
Lepton and jet charge confusion & 0.01 & 0.01 & 0.01 & 0.01 \\
\hline
\end{tabular}


fects due to uncertainties in charge confusion, both for leptons in $q q \ell \nu$ events and jet charges in $q q q q$ events, background normalisation and shape, and technical parameters such as box size and box occupancy are negligible.

As a cross check, a simplified analysis in $\cos \Theta_{\mathrm{w}}$ only is performed using the GENTLE [22] program. Excellent agreement between the shape of the $\cos \Theta_{\mathrm{w}}$ distributions predicted by EXCALIBUR and GENTLE is observed. Detector effects are included by folding the differential cross section in $\cos \Theta_{W}$, calculated by GENTLE as a function of anomalous triple-gauge-boson couplings, with a constant migration matrix relating generated to reconstructed $\cos \Theta_{\mathrm{w}}$ values. The matrix method to incorporate detector effects is extended to all three phase-space angles, then using EXCALIBUR. Within the errors, the same results are obtained with either method.

Assuming all other triple-gauge-boson couplings as given by the Standard Model, the result on $\delta_{Z}$ is:

$$
\begin{aligned}
\delta_{\mathrm{Z}}=g \mathrm{ZWW}-\cot \theta_{\mathrm{W}} & =0.10_{-0.92}^{+1.03}(68 \% \mathrm{CL}) \\
& =0.10_{-1.57}^{+1.76}(95 \% \mathrm{CL}),
\end{aligned}
$$

including systematic errors. The existence of the ZWW vertex is thus established at more than $95 \%$ confidence level. This conclusion is independent of anomalous electromagnetic properties of the $\mathrm{W}$ bo-

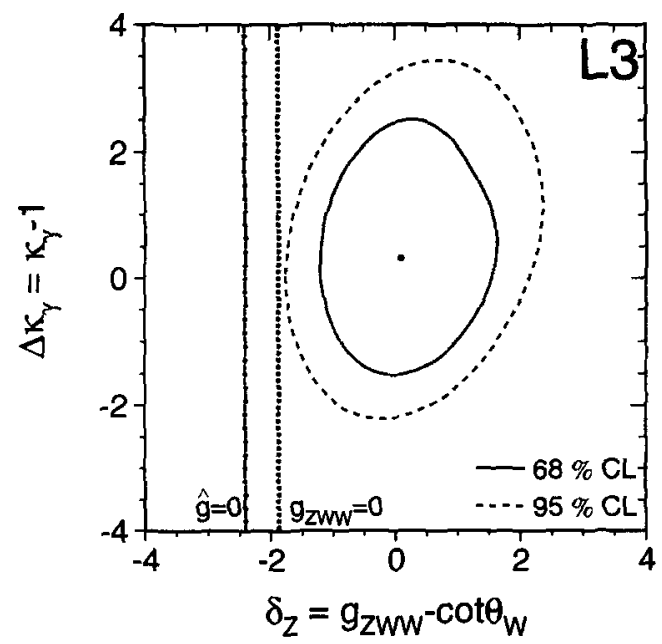

Fig. 5. Contour curves of $68 \%$ and $95 \%$ confidence level in the $\left(\delta_{z}, \Delta \kappa_{\gamma}\right)$ plane are shown as solid and dashed lines. Expectations due to vanishing $\mathrm{ZWW}$ and weak couplings, $g \mathrm{ZWW}=0$ and $\hat{g}=0$, are indicated by the dotted and dashed-dotted lines. son, such as an anomalous contribution $\Delta \kappa_{\gamma}$ to its magnetic moment. Allowing $\Delta \kappa_{\gamma}$ to deviate from zero also, the results are:

$\delta_{Z}=0.09_{-0.94}^{+1.05}(68 \% \mathrm{CL})$

$\Delta \kappa_{\gamma}=0.31_{-1.11}^{+1.81}(68 \% \mathrm{CL})$,

with a correlation coefficient of $-30 \%$ between $\delta_{Z}$ and $\Delta \kappa_{\gamma}$. The constraint $\Delta \kappa_{\mathrm{Z}}=\delta_{z} \tan \theta_{\mathrm{W}}-$ $\Delta \kappa_{\gamma} \tan ^{2} \theta_{\mathrm{W}}$ required by $\mathrm{SU}(2)$ invariance is imposed $[30,3]$. The corresponding contour curves of $68 \%$ and $95 \%$ probability in the $\left(\delta_{Z}, \Delta \kappa_{\gamma}\right)$ plane are shown in Fig. 5. The special cases, $\delta_{Z}=-\cot \theta_{W}$, i.e., vanishing ZWW coupling, and $\delta_{Z}=-\tan \theta_{\mathrm{W}}-\cot \theta_{\mathrm{W}}$, where weak triple-gauge-boson couplings come about only by $y / \mathrm{Z}$ mixing $(\hat{g}=0)$ [31], are excluded by more than $95 \%$ confidence level. For the $\alpha$ triplegauge-boson couplings, the results are:

$$
\begin{aligned}
& \alpha_{\mathrm{W} \Phi}=0.04_{-0.35}^{+0.43}(68 \% \mathrm{CL}) \\
& \alpha_{\mathrm{W}}=0.22_{-0.61}^{+0.59}(68 \% \mathrm{CL}) \\
& \alpha_{\mathrm{B} \Phi}=0.07_{-1.16}^{+1.77}(68 \% \mathrm{CL}),
\end{aligned}
$$

where for the determination of each gauge coupling all others are set to their Standard Model value. In all scenarios good agreement with the Standard Model expectation of $\delta_{Z}=\Delta \kappa_{\gamma}=\alpha_{\mathrm{W} \Phi}=\alpha_{\mathrm{W}}=\alpha_{\mathrm{B} \Phi}=0$ is observed.

\section{Acknowledgements}

We wish to congratulate the CERN accelerator divisions for the successful upgrade of the LEP machine and to express our gratitude for its good performance. We acknowledge with appreciation the effort of all engineers, technicians and support staff who have participated in the construction and maintenance of this experiment. We also thank D. Schildknecht for useful discussions.

\section{References}

[1] S.L. Glashow, Nucl. Phys. 22 (1961) 579;

S. Weinberg, Phys. Rev. Lett. 19 (1967) 1264; A. Salam, in: N. Svartholm (Ed.), Elementary Particle Theory, Stockholm. Almquist and Wiksell, 1968, p. 367. 
[2] Z. Kunszt et al., in: G. Altarelli, T. Sjöstrand, F. Zwirner (Eds.), Physics at LEP 2, Report CERN 96-01, 1996, vol. 1, p. 141.

[3] G. Gounaris et al., in: G. Altarelli, T. Sjöstrand, F. Zwirner (Eds.), Physics at LEP 2, Report CERN 96-01 (1996), vol. 1, p. 525 .

[4] UAl Collaboration, C. Albajar et al., Z. Phys. C 44 (1989) 15;

UA2 Collaboration, J. Alitti et al., Phys. Lett. B 241 (1990) 150; Phys. Lett. B 276 (1992) 354;

CDF Collaboration, F. Abe et al., Phys. Rev. Lett. 65 (1990) 2243; Phys. Rev. D 43 (1991) 2070; Phys. Rev. Lett. 75 (1995) 11; Phys. Rev. D 52 (1995) 4784;

$D \emptyset$ Collaboration, S. Abachi et al., Phys. Rev. Lett. 77 (1996) 3309;

we use the average value and error for the mass of the $W$ boson as listed in: R.M. Barnett et al., Review of Particle Properties, Phys. Rev. D 54 (1996) 1.

[5] UA1 Collaboration, C. Albajar et al., Phys. Lett. B 253 (1991) 503;

UA2 Collaboration, J. Alitti et al., Phys. Lett. B 276 (1992) 365 ;

CDF Collaboration, F. Abe et al., Phys. Rev. Lett. 74 (1995) 341; Phys. Rev. D 52 (1995) 2624;

DØ Collaboration, S. Abachi et al., Phys. Rev. Lett. 75 (1995) 1456;

we use the average value and error for the total width of the W boson as listed in: R.M. Barnett et al., Review of Particle Properties, Phys. Rev. D 54 (1996) 1.

[6] UA2 Collaboration, J. Alitti et al., Phys. Lett. B 277 (1992) 194;

CDF Collaboration, F. Abe et al., Phys. Rev. Lett. 74 (1995) 1936; Phys. Rev. Lett. 74 (1995) 1941; 75 (1995) 1017; 78 (1997) 4536;

DØ Collaboration, S. Abachi et al., Phys. Rev. Lett. 75 (1995) 1023; Phys. Rev. Lett. 75 (1995) 1028, 1034: Phys. Rev. Lett. 77 (1996) 3303; 78 (1997) 3634, 3640;

S. Abachi et al., Studies of gauge boson pair production and trilinear couplings. Fermilab-Pub-97/088-E, hep-ex/ 9704004;

B. Abbott et al., Limits on WWZ and WW $\gamma$ couplings from $p \bar{p} \rightarrow$ evjetjet $X$ events at $\sqrt{s}=1.8 \mathrm{TeV}$, Fermilab-Pub97/136-E, hep-ex/9705010.

[7] L3 Collaboration, M. Acciarri et al., Phys. Lett. B 398 (1997) 223.

[8] L3 Collaboration, M. Acciarri et al., Measurement of W-Pair Cross Sections in $e^{+} e^{-}$Interactions at $\sqrt{s}=172 \mathrm{GeV}$ and W-Decay Branching Fractions, CERN-PPE/97-67.

[9] ALEPH Collaboration, R. Barate et al., Phys. Lett. B 401 (1997) 347;

OPAL Collaboration, K. Ackerstaff et al., Phys. Lett. B 389 (1996) 416.

[10] DELPHI Collaboration, P. Abreu et al., Phys. Lett. B 397 (1997) 158.

[11] L3 Collaboration, M. Acciarri et al., Phys. Lett. B 403 (1997) 168.
[12] OPAL Collaboration, K. Ackerstaff et al., Phys. Lett. B 397 (1997) 147.

[13] L3 Collaboration, B. Adeva et al., Nucl. Instr. and Meth. A 289 (1990) 35;

M. Chemarin et al., Nucl. Instr. and Meth. A 349 (1994) 345; M. Acciarri et al., Nucl. Instr. and Meth. A 351 (1994) 300; G. Basti et al., Nucl. Instr. and Meth. A 374 (1996) 293;

I.C. Brock et al., Nucl. Instr. and Meth. A 381 (1996) 236; A. Adam et al., Nucl. Instr. and Meth. A 383 (1996) 342.

[14] The working group on LEP energy, LEP Energy Calibration in 1996, LEP Energy Group/97-01.

[15] The KORALW version 1.21 is used. M. Skrzypek, S. Jadach, W. Placzek, Z. Wa̧s, Comp. Phys. Comm. 94 (1996) 216; M. Skrzypek, S. Jadach, M. Martinez, W. Placzek, Z. Wạs, Phys. Lett. B 372 (1996) 289.

[16] The HERWIG version 5.9 is used. G. Marchesini, B. Webber, Nucl. Phys. B 310 (1988) 461;

I.G. Knowles, Nucl. Phys. B 310 (1988) 571;

G. Marchesini et al., Comp. Phys. Comm. 67 (1992) 465.

[17] F.A. Berends, R. Kleiss, R. Pittau, Nucl. Phys. B 424 (1994) 308; Nucl. Phys. B 426 (1994) 344; Nucl. Phys. B (Proc. Suppl.) 37 (1994) 163;

R. Kleiss, R. Pittau, Comp. Phys. Comm. 83 (1994) 141:

R. Pittau, Phys. Lett. B 335 (1994) 490.

[18] T. Sjöstrand, PYTHIA 5.7 and JETSET 7.4 Physics and Manual, CERN-TH/7112/93 (1993), revised August, 1995; Comp. Phys. Comm. 82 (1994) 74.

[19] The KORALZ version 4.01 is used. S. Jadach, B.F.L. Ward, Z. Wạs, Comp. Phys. Comm. 79 (1994) 503.

[20] J.H. Field, Phys. Lett. B 323 (1994) 432; J.H. Field, T. Riemann, Comp. Phys. Comm. 94 (1996) 53.

[21] The L3 detector simulation is based on GEANT Version 3.15. R. Brun et al., GEANT 3, CERN-DD/EE/84-1 (Revised), 1987

The GHEISHA program (H. Fesefeldt, RWTH Aachen Report PITHA $85 / 02(1985)$ ) is used to simulate hadronic interactions.

[22] The GENTLE version 2.0 is used. D. Bardin et al., GENTLE/4fan v. 2.0: A Program for the Semi-Analytic Calculation of Predictions for the Process $e^{+} e^{-} \rightarrow 4 f$, DESY 96233, hep-ph/9612409.

[23] D.M. Schmidt, R.J. Morrison, M.S. Witherell, Nucl. Instr. and Meth. A 328 (1993) 547.

[24] G. Gustafson, U. Petterson, P.M. Zerwas, Phys. Lett. B 209 (1988) 90;

T. Sjöstrand, V.A. Khoze, Z. Phys. C 62 (1994) 281; Phys. Rev. Lett. 72 (1994) 28;

E. Accomando, A. Ballestrero, E. Maina, Phys. Lett. B 362 (1995) 141 ;

G. Gustaffson, J. Häkkinen, Z. Phys. C 64 (1994) 659;

L. Lönnblad, Z. Phys. C 70 (1996) 107;

J. Ellis, K. Geiger, Phys. Rev. D 54 (1996) 1967, Signatures of Parton Exogamy in $e^{+} e^{-} \rightarrow W^{+} W^{-} \rightarrow$ hadrons, CERNTH/97-046.

[25] L. Lönnblad, T. Sjöstrand, Phys. Lett. B 351 (1995) 293; S. Jadach, K. Zalewski, W Mass Reconstruction from 
Hadronic Events in LEP-2: Bose Einstein Effect, CERNTH/97-029.

[26] W. Beenakker et al., in: G. Altarelli, T. Sjöstrand, F. Zwirner (Eds.), Physics at LEP 2, Report CERN 96-01, 1996, vol. 1, p. 79.

[27] L3 Collaboration, M. Acciarri et al., Z. Phys. C 62 (1994) 551.
[28] K. Hagiwara, R.D. Peccei, D. Zeppenfeld, K. Hikasa, Nucl Phys. B 282 (1987) 253.

[29] K. Gaemers, G. Gounaris, Z. Phys. C 1 (1979) 259.

[30] M. Bilenky, J.L. Kneur, F.M. Renard, D. Schildknecht, Nuc1. Phys. B 409 (1993) 22; B 419 (1994) 240.

[31] I. Kuss, D. Schildknecht, Phys. Lett. B 383 (1996) 470.

[32] R.D. Field, R.P. Feynman, Nucl. Phys. B 136 (1978) 1. 